\title{
Analysis of Metal Transfer Through Equilibrium Shape of Pendent Drop in GMAW*
}

\author{
By Sang K. Choi**, Kang H. Lee ${ }^{* *}$, Choong D. Yoo** and Tae S. Lee***
}

\begin{abstract}
In this investigation, a new approach based on the energy minimization method is proposed to analyze the mechanism of the metal transfer (globular and spray modes) in the GMAW process. Energies due to the surface tension, gravity, electromagnetic and drag forces exerted on the pendant drop are minimized and the shape of molten drop in the equilibrium state is calculated without presumed geometry. The energy due to the electromagnetic force is derived using the Maxwell stress. Effects of welding conditions on the shape of the pendant molten drop are analyzed. Results show that the proposed approach can be applied to both globular and spray modes. When compared with other approaches such as force balance and pinch instability models, the results of proposed approach show similar trends and predict the available experimental results more accurately.
\end{abstract}

Key words: Metal Transfer Mode (Globular and Spray Modes), Energy Minimization Method, Molten Drop Shape, Welding Condition, Maxwell Stress

\section{Introduction}

As the metal transfer in the GMAW affects the weld quality and productivity, it has been investigated to figure out the mechanism of molten drop formation and detachment at various welding conditions ${ }^{1)}$. When the molten drop transfers from the electrode to workpiece through the arc, the metal transfer is classified into the globular and spray modes. The static force balance model and pinch instability theory have been most widely used to analyze the metal transfer in the globular and spray modes, respectively.

The force balance model ${ }^{2,3)}$ assumes that the molten drop is a sphere with its diameter larger than the electrode diameter. Attaching and detaching forces in the axial direction due to the surface tension, gravity, electromagnetic and drag forces are calculated, and the molten drop detaches when the detaching force becomes larger than the attaching force. The pinch instability model ${ }^{4,5)}$ utilizes the Rayleigh's instability theory that the liquid column breaks up at the critical wavelength. The geometry of the molten drop is assumed to be a cylinder.

While there is a model that includes the molten drop geometry, effects of the surface tension and gravity are considered because of computational complexity ${ }^{6}$. No consideration was given for the effect of the electromagnetic force. Recently, an attempt was made to model the metal transfer using the energy minimization method $^{7}$. This model predicts the molten drop geometry and shows relatively good agreement with the experimental data. However, the geometry is rather simplified due to difficulty in calculating the electromagnetic energy term.
The model proposed in this work is modified to overcome the shortcomings of the previous energy model. Energies due to the surface tension, gravity, electromagnetic and drag forces are calculated with no presumed geometry. The Maxwell stress is utilized to derive the energy due to the electromagnetic force. The system energy is minimized to obtain the molten drop geometry in the equilibrium state and its detaching conditions. Effects of welding conditions on the metal transfer are explained. When compared with other models, results of the model show better agreements with the available experimental data ${ }^{8)}$, which demonstrates the validity of the present model.

\section{Modeling}

\subsection{Formulation}

The system energy exerted on the molten drop in the equilibrium state can be described as the sum of energies due to the surface tension, gravity, electromagnetic and drag forces:

$$
\mathrm{E}_{\mathrm{sys}}=\mathrm{E}_{\mathrm{st}}+\mathrm{E}_{\mathrm{g}}+\mathrm{E}_{\mathrm{em}}+\mathrm{E}_{\mathrm{d}}
$$

In order to calculate the energies, it is necessary to define the molten drop geometry and displacement within the molten drop. The geometry of the molten drop is assumed in this work to consist of tapered cylindrical slabs as shown in Fig. 1. When the tapered slabs are deformed from initial circular slabs, the displacement in the $\mathrm{i}$-th slab should satisfy the volume constant and boundary conditions. The displacements within the deformed $i$-th slab in the $r$ and $z$ directions are assumed in the polynomial form as:

$$
u_{r}^{(1)}=r\left[1-\frac{a_{0} L_{1}}{\left(a_{1}-a_{1-1}\right) z+a_{1-1} \bar{L}_{1}}\right]
$$

\footnotetext{
* Received : 17 April 1995.

** Dept. of Automation \& Design Eng., Korea Advanced Inst. of Sci. \& Tech. (KAIST), Seoul, Korea

*** Dept. of Mech. Eng., Sogang Univ., Seoul, Korea
} 


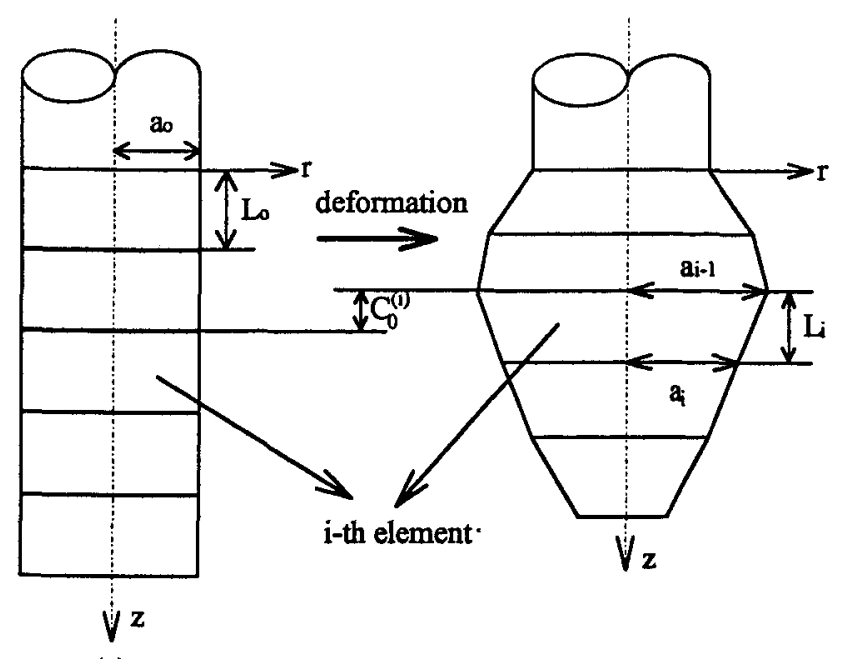

(a)

(b)

Fig. 1 Discretization of molten tip by slabs. (a) initial shape, (b) deformed shape.

$$
\begin{aligned}
& \mathrm{u}_{2}^{(1)}=\mathrm{C}_{0}^{(1)}+\left(\frac{\mathrm{L}_{1}-\mathrm{L}_{0}}{\mathrm{~L}_{1}}\right) z \\
& \mathrm{C}_{0}^{(1)}=\sum_{\mathrm{k}=1}^{\mathrm{i}-1} \mathrm{~L}_{\mathrm{k}}-(\mathrm{i}-1) \mathrm{L}_{0}
\end{aligned}
$$

where $a_{0}$ is the electrode radius, $a_{1}$ and $L_{1}$ are radius and length of the $\mathrm{i}$-th slab, respectively. To satisfy the volume constant condition, the length of $i$-th slab becomes,

$$
\mathrm{L}_{1}=\frac{3 \mathrm{a}_{0}^{2} \mathrm{~L}_{0}}{\mathrm{a}_{\mathrm{i}}^{2}+\mathrm{a}_{1} \mathrm{a}_{1-1}+\mathrm{a}_{\mathrm{i}-1}^{2}}
$$

We note that the displacement derived in this work is not unique: other trial functions can be used if they satisfy the volume constant and boundary conditions.

As the system energy is expressed as the function of unknown radii $a_{1}$, the molten drop geometry can be determined by minimizing the system energy as:

$$
\frac{\partial \mathrm{E}_{\text {sys }}}{\partial \mathrm{a}_{1}}=0, \quad \mathrm{i}=1, \cdots, \mathrm{n}
$$

where $\mathrm{n}$ is the number of the slabs. Above $\mathrm{n}$ equations are solved simultaneously using Newton-Raphson method $^{\text {). }}$

\subsection{Calculation of Energies}

When the material properties such as the surface tension and mass density are assumed to be constant, the energy due to the surface tension is,

$$
\mathrm{E}_{\mathrm{st}}=\int_{\mathrm{A}} \sigma \mathrm{dA}=\sigma \sum_{\mathrm{i}=1}^{\mathrm{n}} \mathrm{A}^{(1)}
$$

where $\sigma$ denotes the surface tension coefficient, $\mathrm{A}^{(1)}$ the surface area of the $\mathrm{i}$-th element. As for the energy due to gravity, it decreases as the displacement increases in the $z$-direction, that is expressed as:

$$
E_{\mathrm{g}}=-\int_{\mathrm{V}} \rho \overrightarrow{\mathrm{g}} \cdot \overrightarrow{\mathrm{u}} \mathrm{dV}=-\rho \mathrm{g} \sum_{\mathrm{i}=1}^{\mathrm{n}} \mathrm{u}_{z}^{(1)} \mathrm{V}^{(1)}
$$

where $\rho$ denotes the mass density, $\vec{g}$ the gravity $(9.8 \mathrm{~m} /$ $\sec ^{2}$ ) and $V^{(i)}$ the volume of the i-th slab.

Derivation of the electromagnetic energy is one of the important features of this model. The electromagnetic

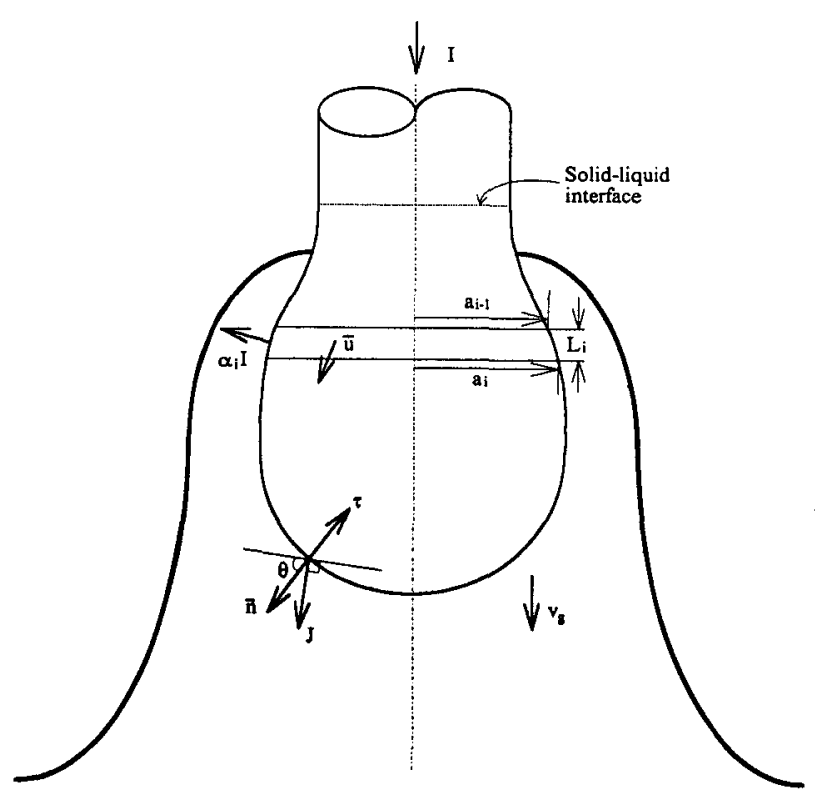

Fig. 2 Notations used in electromagnetic and drag forces.

force is the body force generated by the welding current and self-induced magnetic field:

$$
\int_{\mathrm{V}} \overrightarrow{\mathrm{F}}_{\mathrm{m}} \mathrm{dV}=\int_{\mathrm{V}} \overrightarrow{\mathrm{J}} \times \overrightarrow{\mathrm{B}} \mathrm{dV}
$$

where $\vec{J}$ and $\vec{B}$ are the current density and magnetic flux density, respectively. Because it is difficult to find the current density in the tapered cylinder which satisfies the continuity and boundary conditions, Maxwell stress in utilized instead of deriving the current density directly. The volume integral in Eqn(9) can be converted into the surface integral:

$$
\int_{\mathrm{V}} \overrightarrow{\mathrm{F}}_{\mathrm{m}} \mathrm{dV}=\int_{\mathrm{S}}\left[\frac{\mathrm{B}^{2}}{2 \mu_{0}}\right](-\overrightarrow{\mathrm{n}}) \mathrm{dS}+\int_{\mathrm{S}}\left[\frac{\mathrm{B}^{2}}{\mu_{0}}\right] \overrightarrow{\mathrm{B}} \cos \theta \mathrm{dS}
$$

where $\vec{n}$ is the surface normal vector and $\theta$ is the angle between $\vec{B}$ and $\vec{n}$ as shown in Fig. 2. When the current is assumed to flow to the arc in the normal direction to the molten drop surface $\left(\theta=90^{\circ}\right)$, the last term of Eqn(10) is eliminated ${ }^{2}$, and the electromagnetic energy is derived using the Maxwell stress, $\tau$, as:

$$
\begin{aligned}
& E_{\mathrm{em}}=-\int_{\mathrm{S}} \tau \overrightarrow{\mathrm{n}} \cdot \overrightarrow{\mathrm{u}} \mathrm{dS} \\
& \tau=-\frac{\mathrm{B}^{2}}{2 \mu_{0}}
\end{aligned}
$$

When the $\mathrm{i}$-th slab is covered with the arc, the effect of current flow to the arc is considered using the parameter $\alpha_{1}$ that represents the ratio of the leaking current through the surface of $\mathrm{i}$-th slab to the total current I (i. e., $\left.\sum_{i=1}^{\mathrm{n}} \alpha_{1}=1.0\right)$, as shown in Fig. 2 . The energy due to the electromagnetic force consist of $\mathrm{r}$ and $\mathrm{z}$ components, and they are derived as:

$$
\begin{aligned}
& E_{\text {em }}=E_{\text {em }, \mathrm{r}}+E_{\text {em }, z} \\
& E_{\text {em, }, \mathrm{r}}=-\frac{\mu_{0} I^{2}}{4 \pi} \sum_{\mathrm{i}=1}^{n}\left[( 1 - \sum _ { \mathrm { k } = 1 } ^ { 1 - 1 } \alpha _ { \mathrm { k } } - \frac { \alpha _ { \mathrm { i } } } { 2 } ) ^ { 2 } \left\{\mathrm{~L}_{1}+\right.\right. \\
& \left.\left.\frac{\mathrm{a}_{0} \mathrm{~L}_{1}}{\mathrm{a}_{1}-\mathrm{a}_{1-1}} \ln \left(\frac{\mathrm{a}_{1-1}}{\mathrm{a}_{\mathrm{i}}}\right)\right\}\right]
\end{aligned}
$$




$$
\begin{aligned}
\mathrm{E}_{\mathrm{em}, 2}=\frac{\mu_{0} \mathrm{I}^{2}}{4 \pi} \sum_{\mathrm{i}=1}^{\mathrm{n}}\left[( 1 - \sum _ { \mathrm { k } = 1 } ^ { 1 - 1 } \alpha _ { \mathrm { k } } - \frac { \alpha _ { \mathrm { i } } } { 2 } ) ^ { 2 } \left\{\mathrm{C}_{0}^{(1)}-\right.\right. \\
\left.\left.\frac{\mathrm{a}_{\mathrm{i}-1}\left(\mathrm{~L}_{\mathrm{i}}-\mathrm{L}_{0}\right)}{\mathrm{a}_{1}-\mathrm{a}_{\mathrm{i}-1}} \ln \left(\frac{\mathrm{a}_{1-1}}{\mathrm{a}_{\mathrm{i}}}\right)-\mathrm{L}_{\mathrm{i}}+\mathrm{L}_{0}\right\}\right]
\end{aligned}
$$

Using the Maxwell stress, the electromagnetic energy can be derived without deriving the current density within the tapered slab. It becomes also possible to simulate various forms of current distribution by adjusting the values of $\alpha_{1}$ 's.

As the drag force decreases the system energy, the energy due to the drag force is described as:

$$
\mathrm{E}_{\mathrm{d}}=-\frac{\mathrm{C}_{\mathrm{d}} \rho_{\mathrm{g}} \mathrm{v}_{\mathrm{g}}^{2}}{2} \int_{\mathrm{A}_{\mathrm{p}}} \mathrm{u}_{\mathrm{z}} \mathrm{d} \mathrm{A}_{\mathrm{p}}=-\frac{\mathrm{C}_{\mathrm{d}} \rho_{\mathrm{g}} \mathrm{v}_{\mathrm{g}}^{2}}{2} \sum_{\mathrm{l}=1}^{\mathrm{n}} \mathrm{u}_{\mathrm{z}}^{(1)} \mathrm{A}_{\mathrm{p}}^{(1)}(16)
$$

where $\mathrm{C}_{\mathrm{d}}$ denotes the drag coefficient, $\rho_{g}$ the gas density, $\mathrm{V}_{\mathrm{g}}$ the gas velocity including the plasma jet velocity and $A_{p}$ the projected area. The gas velocity ${ }^{5)}$ and projected area of $\mathrm{i}$-th slab are expressed as:

$$
\begin{aligned}
\mathrm{v}_{\mathrm{g}} & =20.4+0.37 \mathrm{I}+0.0004344 \mathrm{I}^{2} \\
\mathrm{~A}_{\mathrm{p}}^{(1)} & =\pi\left(\mathrm{a}_{1}^{2}-\mathrm{a}_{1-1}^{2}\right), \text { if } \mathrm{a}_{1}>\mathrm{a}_{1-1} \\
& =0, \text { otherwise }
\end{aligned}
$$

The drag coefficient of the sphere ${ }^{10)}$ is used for simplification. It is based on the fact that the shape of molten drop is somewhat similar to that of the sphere in the globular mode, and the energy due to the drag force is neglected in the spray mode because the projected area becomes zero as described in Eqn(18).

\section{Results and Discussion}

Effects of welding conditions such as the welding current, molten drop volume, arc covered area and current density distribution on the molten drop geometry and detachment are predicted using the model. Material properties of the steel electrode with $1.6 \mathrm{~mm}$ diameter and argon shielding gas used for calculation are listed in Table $1^{5}$. The molten drop volume is

Table 1 Material properties.

\begin{tabular}{|c|c|}
\hline Parameter & Value \\
\hline$\rho$ & $7800\left(\mathrm{~kg} / \mathrm{m}^{3}\right)$ \\
\hline$\gamma$ & $1.2(\mathrm{~N} / \mathrm{m})$ \\
\hline$\dot{\rho}_{\mathrm{g}}$ & \\
\hline (argon) & $0.06\left(\mathrm{~kg} / \mathrm{m}^{3}\right)$ \\
\hline$\mu$ & $2.56 \times 10^{-4}(\mathrm{~kg} / \mathrm{ms})$ \\
\hline $\begin{array}{c}\text { (argon, 8000K) } \\
\mu_{0}\end{array}$ & $4 \pi \times 10^{-7}\left(\mathrm{~N} / \mathrm{A}^{2}\right)$ \\
\hline
\end{tabular}

expressed as the initial molten electrode length that is divided by the 16 slabs.

In order to ensure numerical convergence, the equilibrium shape of the molten drop due to the surface tension energy only is calculated from a cylinder with given initial drop length. The effects of gravity, electromagnetic and drag forces are added sequentially to the equilibrium shape due to the surface tension energy, as shown in Fig. 3. The welding current increases gradually to obtain the stable solutions until the solution diverges.

The effects of various forces on the shape of molten drop are shown in Fig. 3. When only the surface tension is exerted on the molten drop, the shape is similar to the sphere. Adding the effects of gravity, electromagnetic and drag forces, the molten drop elongates with decrease in radius. In this case, the entire molten drop surface is assumed to be uniformly covered by the arc. Whan initial length is $0.5 \mathrm{~mm}$ and welding current is 322 A (Fig. 3a), the effect of gravity is negligible and that of the electromagnetic force is dominant. This configuration of the molten drop shape represents spray mode. In the spray mode, the effect of the drag force is not

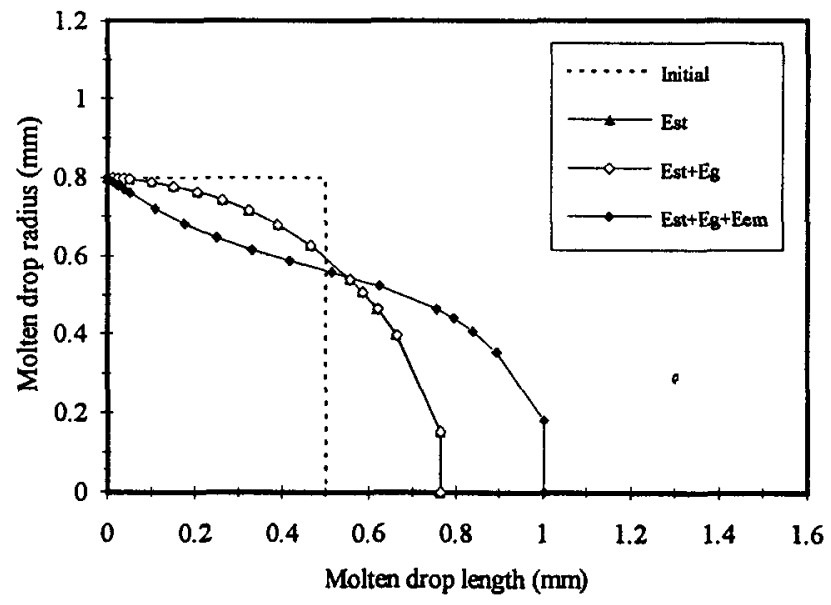

(a)

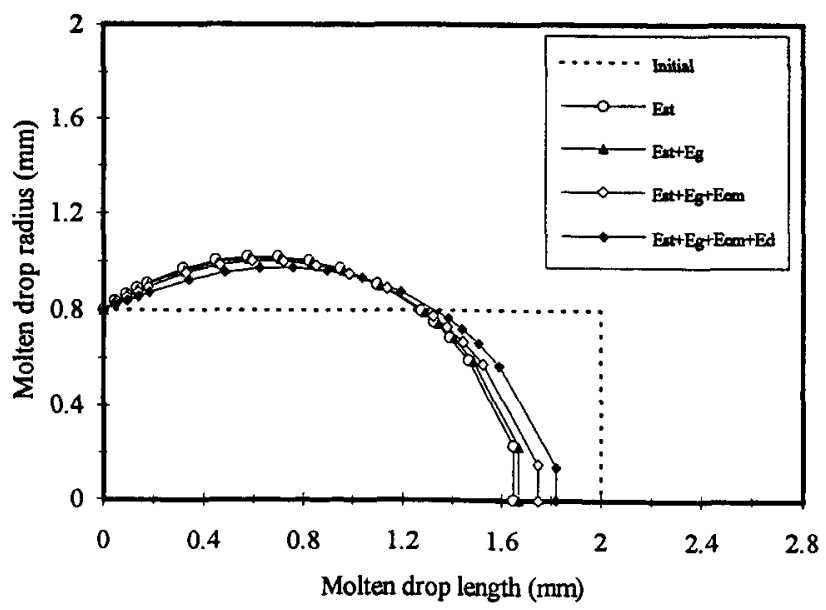

(b)

Fig. 3 Comparison of molten tip shape for various acting forces. (a) initial length: $0.5 \mathrm{~mm}$ at $322 \mathrm{~A}$, (b) initial length : $2 \mathrm{~mm}$ at $201 \mathrm{~A}$. 


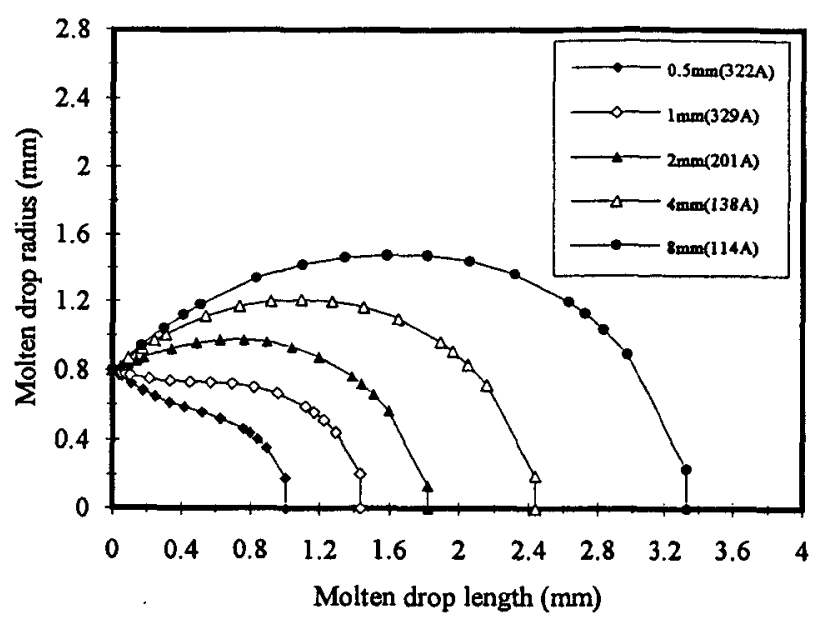

Fig. 4 Comparison of molten tip shape for various initial volume.

considered because of no projected area to the flow of shield gas. However, it should be noted that the plasma stream in the spray mode affects the metal transfer which is difficult to consider quantitatively in the present model. When initial length is $2 \mathrm{~mm}$ and welding current is 201 A (Fig. 3b), the effect of gravity becomes more pronounced but it still has minor effects on the shape when compared with the other forces. The effects of the electromagnetic and drag forces are dominant, and the configuration represents the globular mode.

When the entire molten drop surface is covered uniformly by the arc, the relation between the molten drop volume, welding current and molten drop shape is illustrated in Fig. 4. For each molten drop volume, the geometry is calculated at the critical current above which the solution of Eqn( 6 ) diverges. It appears that the equilibrium is violated above the critical current and instability starts to occur. It is speculated that molten drop detaches from the electrode above the critical current. With the initial molten drop length of $0.5,1,2$, 4 , and $8 \mathrm{~mm}$, corresponding critical currents are 322,329 , 201, 138, and $114 \mathrm{~A}$, respectively, which illustrates the general trend that the current increases with decrease in the molten drop volume. Although the reason is not clearly understood, it is noted that the values of the critical current vary between 320 and $330 \mathrm{~A}$ when the initial drop length becomes smaller than $1 \mathrm{~mm}$. When the initial length is below $1 \mathrm{~mm}$, the radius of the molten drop becomes smaller than that of the electrode, and the metal transfer occurs in the spray mode. As the molten drop grows larger, the critical current becomes lower and the molten drop geometry demonstrates the globular mode. It agrees with the widely known fact that the spray mode occurs at higher current above the transition current.

The arc covered area, that is defined to be the surface of slabs covered by the arc, is known to have relation with the shielding gas composition and metal transfer mode. It has been observed that while the molten drop

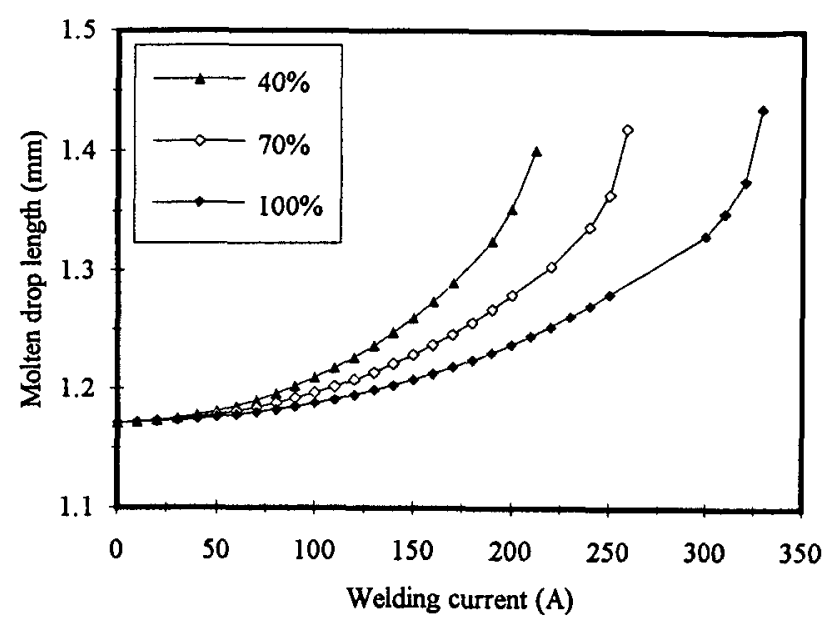

Fig. 5 Effects of arc covered area.

is fully covered by the arc in the spray mode, only bottom part of the molten drop is covered by the arc in the globular mode ${ }^{8}$. The effect of the arc covered area with uniform current density is shown in Fig. 5 when the initial molten drop length is $1 \mathrm{~mm}$. The percentage in the figure represents initial values of the ratio of the surface area of the slabs uniformly covered by the arc to the total surface area of the molten drop of the initial cylindrical shape (Fig. 1a). Initial input values of the arc covered area ratio are 40,70 and $100 \%$. When the equilibrium shapes are calculated using different welding currents, the surface area of the molten drop changes and the ratio of arc covered area varies within the range of approximately $10 \%$. At the same current, the molten drop length increases when the arc covered area decreases. It appears that the high current density within the slabs which are not covered by the arc produces high pinch force, which elongates the molten drop more. However, the critical current increases as the arc covered area increases. Therefore, the spray mode occurs when the arc covered area increases, which agrees with the experimental observation.

As the current density distribution on the surface of the molten drop is not clearly known, effects of the constant (or uniform) and linear distribution of the current density are calculated and shown in Fig. 6. In the linear case, the current density is increased linearly from zero current density at the solid-liquid interface. As smaller amount of the welding current flows to the arc at the upper part of the molten drop in the linear case, it is speculated that there exists higher current within the slab which generates higher pinch force and results in more elongation than the constant current density case. The critical current increases with the constant current density distribution that is similar to the results of arc covered area (Fig. 5).

Results of the energy model are compared with those of the force balance and pinch instability models, and available experimental data ${ }^{8}$ as shown in Fig. 7. For the energy and force balance models, the entire molten drop 


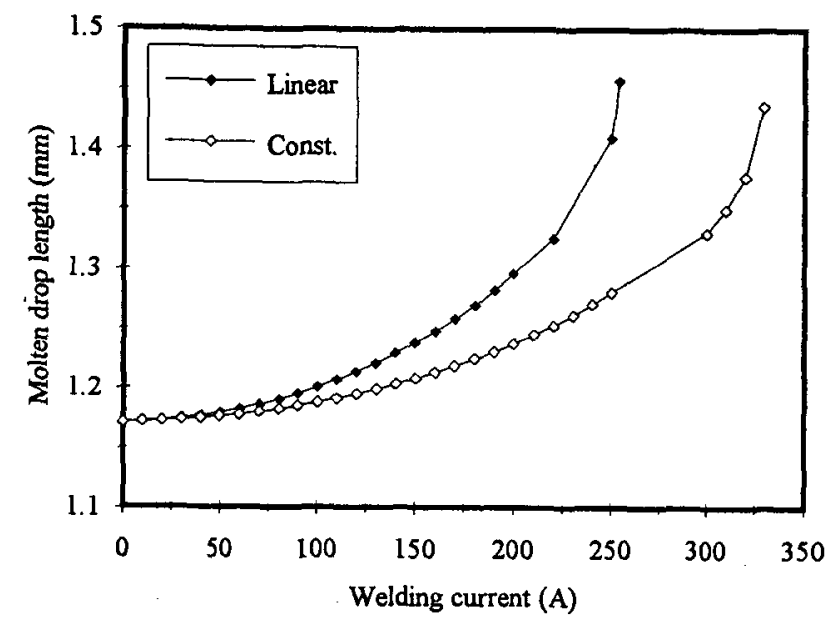

Fig. 6 Effect of current density distribution.

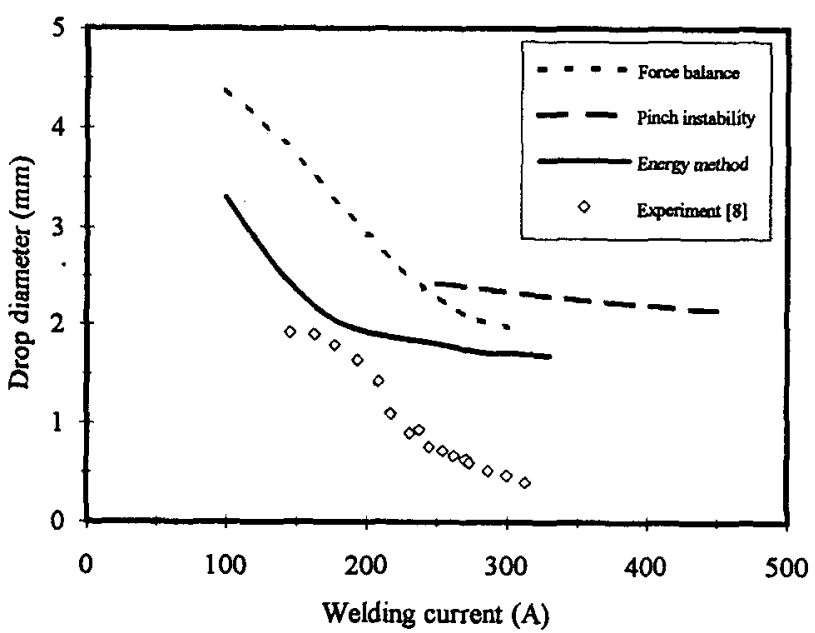

Fig. 7 Comparison with other models and experimental data.

is fully covered by the arc. The molten drop volume is converted to the diameter of the sphere for comparison. Although the converted drop diameter appears to be larger than that of electrode at about $300 \mathrm{~A}$ in the plot, the predicted molten drop shape by the energy model represents spray mode (Fig.4). The energy model shows similar trends to other models such that the drop diameter decreases with current increase. When compared with the experimental results, the energy model predicts more accurately. It appears that the energy model can predict the metal transfer more accurately by considering the molten drop geometry. The disadvantages of the energy model are that the welding current cannot be increased above the critical currents and that the effect of plasma jet in the spray mode cannot be considered. In order to overcome these shortcomings, further study on the dynamics and instability should be carried out.

\section{Conclusions}

A model is developed using the energy minimization method that can predict the molten drop geometry and detachment. Results of this work lead to the following conclusions :

(1) Both globular and spray modes can be analyzed using the energy model that includes the effects of the surface tension, gravity, electromagnetic and drag forces. In both modes, the effect of the electromagnetic force is dominant compared with that of gravity.

(2) When the molten drop volume decreases and the arc covered area increases, the current can be increased above the transition current, and the predicted geometry of the molten drop shows that the spray mode occurs. Above the critical current, the equilibrium is violated and the molten drop appears to detach from the electrode.

(3) The energy model shows similar trends with other models and predicts the experimental results more accurately.

\section{References}

1) Lancaster, I.F., 1985, The physics of welding, Pergamon Press.

2) Greene, W.J., 1960, "An analysis of transfer in gas-shielded welding arcs", Trans. Amer. Inst. Elec. Engr., Vol. 79, pp. 194 $-203$

3) Amson, J.C., 1965, "Lorentz force in the molten tip of an arc electrode", Brit. J. Appl. Phys., Vol. 16, pp. 1169-1179.

4) Allum, C.J., 1985, "Metal transfer in arc welding as a varicose instability", J. Applied Physics, Vol. 18, pp. 1447-1468.

5) Rhee, S.H., and Kannatey-Asibu Jr., E., 1991, "Analysis of arc pressure effect on metal transfer in gas-metal arc welding”, J. Appl. Phys., Vol. 70, pp. 5068-5075.

6) Cram, L.E.L. 1984, "A Numerical Model of Droplet Formulation" in Computational Techniques and Applications, Ed. J. Noye and C. Fletcher. Elsevie Science Publishers B.V. North-Holland.

7) Joo, T.M., 1994, "Analysis of Molten Tip Shape by Energy Method in GMA Process", MS Thesis, Korea Advanced Institute of Science \& Technology (KAIST)

8) Kim, Y.S., 1989, "Metal transfer in gas metal arc welding", Ph. D Thesis, M.I.T.

9) Press, W.H., Flannery, B.P., Teukolsky S.A., and Vetterling W.T., 1988, Numerical recipes in C, Cambridge Univ. Press.

10) White, F.M., 1974, Viscous Flow, McGraw-Hill, New York. 